\title{
Postoperative exercise training is associated with reduced respiratory infection rates and early discharge: A case-control study ${ }^{\text {hi }}$
}

\author{
N.R. Bhatt ${ }^{a}$, G. Sheridan ${ }^{a}$, M. Connolly ${ }^{a}$, S. Kelly ${ }^{a}$, A. Gillis ${ }^{a}$, \\ K.C. Conlon ${ }^{a}$, S. Lane ${ }^{b}$, E. Shanahan ${ }^{c}$, P.F. Ridgway ${ }^{a, *}$ \\ a Professorial Surgical Unit, University of Dublin, Trinity College, at the Adelaide and Meath Hospital, Tallaght, \\ Dublin 24, Ireland \\ ${ }^{\mathrm{b}}$ Department of Respiratory Medicine, Adelaide and Meath Hospital, Tallaght, Dublin 24, Ireland \\ ${ }^{\mathrm{c}}$ Department of Anaesthesia, Adelaide and Meath Hospital, Tallaght, Dublin 24, Ireland
}

\section{A R T I C L E I N F O}

\section{Article history:}

Received 19 May 2015

Received in revised form

7 July 2015

Accepted 9 July 2015

Available online $\mathrm{xxx}$

Keywords:

Postoperative complications

Aerobic exercise

Respiratory tract infection

Length of stay

\begin{abstract}
A B S T R A C T
Introduction: Pulmonary complications are a significant cause of morbidity, mortality and increased hospital stay following complex abdominal surgery. We investigated whether postoperative early aerobic activity with a pedal exerciser reduced respiratory morbidity and length of stay and improved pulmonary function.

Methods: A prospective case-control study on 30 cases and 30 case matched controls aged 18 years or more who underwent major surgery was conducted. Controls were case-mix matched prospectively from a similar general surgical service not utilising postoperative exercising. Thirty consecutive cases were started on a twice-daily aerobic exercise program with pedal exerciser post-operatively day 2 or from when sitting independently. Primary outcome measures were respiratory tract infection (RTI), deep vein thrombosis (DVT) or pulmonary embolus (PE). Secondary outcome measure was subjective breathlessness and Length of Stay (LOS) postoperatively.

Results: The rate of RTI was only $16.6 \%$ in the exercise group and $43.3 \%$ in the control group $(\mathrm{P}=0.024)$. None of the cases or controls suffered from a DVT or PE. Median postoperative length of stay in the control group was $11 \pm 7.5$ days whereas in the cases it was $8.5 \pm 5.00$ days $(\mathrm{P}=0.049)$. The Borg subjective breathlessness score in the cases group showed a decline in the subjective breathlessness on postoperative day $4(\mathrm{P}=0.002)$.

Conclusions: Early aerobic activity with a pedal exerciser halves the rate of postoperative RTI and postoperative hospital stay after complex abdominal surgery. Subjective breathlessness was also reduced with the use of pedal exerciser, signifying potential to improve exercise endurance in the postoperative patient.
\end{abstract}

(c) 2015 Royal College of Surgeons of Edinburgh (Scottish charity number SC005317) and Royal College of Surgeons in Ireland. Published by Elsevier Ltd. All rights reserved.

\footnotetext{
Note: This article was presented at the Surgical Medal Session in the Association of Surgeons in Training (ASIT) conference in Belfast, Ireland.

* Corresponding author. Department of Surgery, Adelaide and Meath Hospital, Tallaght, Dublin 24, Ireland. Tel.: +353 14142211.

E-mail address: ridgwayp@tcd.ie (P.F. Ridgway). http://dx.doi.org/10.1016/j.surge.2015.07.003

1479-666X/@ 2015 Royal College of Surgeons of Edinburgh (Scottish charity number SC005317) and Royal College of Surgeons in Ireland. Published by Elsevier Ltd. All rights reserved.
} 


\section{Introduction}

Pulmonary function is commonly negatively affected by surgery, particularly in patients who have undergone chest or upper abdominal surgery. This occasions a decrease in total pulmonary capacity, functional pulmonary volumes and a parallel decrease in $\mathrm{PaO}_{2}{ }^{1}$. These functional alterations are implicated in the development of Postoperative Pulmonary Complications (PPC), which are a significant cause of morbidity and mortality following surgery. ${ }^{2}$ Postoperative Pulmonary Complications (PPC), are seen in up to $40 \%$ of patients. ${ }^{3,4}$ Lung expansion techniques (such as deep breathing exercises, incentive spirometry and continuous positive airways pressure) may help reduce PPC but have largely fallen out of favour. ${ }^{5}$ This is in part due to the fact that no modality is proven superior, and combined modalities do not seem to provide additional risk reduction. ${ }^{6}$

Our hypothesis was that early aerobic activity with a foot pedal exerciser might produce a significant reduction in respiratory morbidity rates or improvement in measured pulmonary function. A foot pedal exerciser is a portable stationary bike, which allows the patient to perform aerobic activity while sitting comfortably in a chair. Hence, the exercise programme can be started soon after surgery (postoperative day 2) if the patient can sit out.

\section{Methods}

\section{Study design}

This was a prospective case control study on 30 cases and 30 controls to investigate if early post-operative exercise produces a significant reduction in respiratory tract infection rates and a significant improvement in patient pulmonary function. Ethics approval was given by the Institutional Ethics Committee (Tallaght Hospital/St. James's Hospital Joint Research Ethics Committee, 2011). Informed consent was taken from all the patients participating in the study.

A pragmatic assignment design was employed. Clinical cases and controls of surgical complexity Level $2^{7}$ or above were selected by a member of the surgical team (not the operating surgeon) as per the inclusion criteria. The cases were consecutively selected from the waiting list of case-mix appropriate post-operative patients in the general surgical service. Controls were case mix matched prospectively from the same general surgical service not utilising postoperative exercising. Where suitable matches were not present, the controls were allowed lesser or similar surgical complexity. Both cases and controls were managed by two surgical consultants in the same institution with the same trainee teams. Owing to the complexity of the surgeries, all cases and controls were operated by the consultant surgeon or a senior trainee under direct consultant supervision. The clinical teams weren't blinded but were unaware as to which patient would undergo the exercise program at the time of surgery. Anaesthesia was unaltered with practitioners and amount of analgesia, muscle relaxants and reversal were given as they deem fit. Postoperative care was unaltered apart from the addition of pedal exerciser in cases.

The surgeries performed on the subjects were classified into validated Levels of Complexity ${ }^{7}$ depending on (Details: Appendix 1 and 2):

1) The risk to the patient independent of anaesthesia (minimal, moderate or major)

2) Potential Blood loss during surgery (none, $<500 \mathrm{cc}$, $500-1500 \mathrm{cc}$ or $>1500 \mathrm{cc}$ )

3) Invasiveness of the procedure (minimal, moderate or high)

4) Postoperative ICU stay (yes/no)

The American Society of Anaesthetists (ASA) grading was recorded for all cases and controls.

\section{Setting and intervention model}

The exercise program took place in a tertiary level university teaching hospital. A pedal exerciser (Drive Medical, New York, USA) (Fig. 1) was used for the exercise program. It is a portable mini exercise bike, whose resistance level can be changed to offer a more challenging or less challenging workout.

\section{Exercise program}

The cases that were selected were started on an exercise program with pedal exerciser from post-op day 2 or when they were able to sit out (whichever was sooner). The immediate outcome of the exercise was assessed utilising Borg scale. The Borg dyspnoea score is a categorical scale, ${ }^{8,9}$ which is widely used in the field of respiratory rehabilitation as an instrument to prescribe workload during muscle training sessions and the clinical significance of the rehabilitation outcome (in terms of perceived dyspnoea during physical exercise) has been validated. It has been used in numerous studies in COPD patients and emphysematous patients as an outcome measure. A

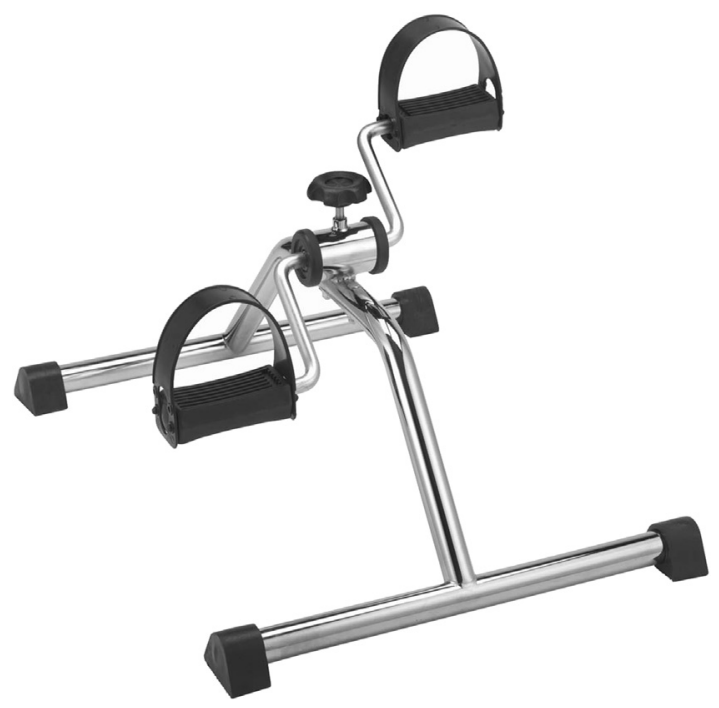

Fig. 1 - The exercise bike used for our study (Drive Medical, NY, USA). 
variation of 2 units with respect to the baseline is associated with a sensation of perceived improvement. ${ }^{10}$

Before the study the patients were familiarised with the Borg scale, and its end points included zero for the absence of dyspnoea and 10 " the most severe level of dyspnoea you could imagine or have ever undergone". By pointing to the scale, the patients perceived dyspnoea at rest and at the end point of the exercise. ${ }^{8}$

The exercise program was done twice daily: morning and evening; and continued till the patients reached one of the following:

- $10 \mathrm{~min}$ on the pedal exerciser

- 3 on Borg breathlessness scale (moderate breathlessness)

$-\mathrm{RR} \geq 35$

- $\geq 70 \%$ maximum heart rate

The level of resistance on the pedal exerciser was set at 0 and each patient was to have a standardised chair position.

The patients were supervised directly by a team member during the exercise program. The parameters noted before and after the exercise program were Borg breathlessness scale, respiratory rate, heart rate, oximetry, total revolutions, exercise time tolerated if it was less than $10 \mathrm{~min}$.

The control group received standard respiratory treatment if required as per hospital protocols. However they did not undergo any prophylactic respiratory rehabilitation, any respiratory intervention (only when necessary) in the control group was only as a treatment measure.

\section{Participants}

\section{Inclusion criteria}

The patient population included all patients above the age of 18 years who had undergone major surgery of Surgical Complexity two or more and were able to sit out on postoperative day two.

A Resting $\mathrm{PaO}_{2} \geq 93 \%$, Supplemental $\mathrm{O}_{2}$ requirement $\leq 28 \%$, LVEF $\geq 40 \%$ and normal motor function lower limbs were also taken as inclusion criteria.

\section{Exclusion criteria}

If the patients had previous Myocardial Infarction/Percutaneous Coronary Intervention or known ischaemic heart disease, diabetes, impaired cognition/confusion or back/lower limb musculoskeletal/neurological pathology impairing ability to sit out or pedal, they were excluded.

The study was approved by our institutional ethics committee, and informed consent was obtained from the patients.

\section{Outcomes}

The outcomes were divided into primary and secondary.

The primary outcome included measured Respiratory Tract Infection (RTI), which was defined as:

1. New or changed CXR appearance suggestive of infection and $>2$ of the following:

1. Post-op unexplained raised tympanic temperature $>38.0^{\circ} \mathrm{C}$,

2. WCC elevation $>12,000$,
3. The development of a productive cough sputum,

4. Patient treated with antibiotics directed for a RTI (antibiotics directed for other infections were not considered).

Deep Vein Thrombosis (DVT) or Pulmonary Embolus (PE) as identified by clinical or radiological imaging: ultrasound, CTPA or V/Q scan was also included in the primary outcome.

The secondary outcomes included measuring subjective breathlessness Borg score before exercise and after exercise from day 2 to day 4 . The postoperative length of stay (LOS) was also included as a secondary outcome.

As a sub analysis we also looked at preoperative hospital stay of more than one day in both cases and controls and if this was related to increased rate of respiratory tract infections.

\section{Statistical analysis}

Normality testing of each variable was done using the Kolmogorov-Smirnov and Shapiro-Wilk tests and further tests were applied as appropriate. The unpaired t test was used to compare age distribution between the two groups. The Mann-Whitney U test was used to compare the type of surgeries and ASA grades between the two groups. The Chi Square test was used to compare proportions of Male: Female, the smoking status, and the administration of epidural analgesia between the two groups. The Chi Square test was also used to compare the primary outcomes between the cases and the controls. The Fischer's exact test was used to compare primary outcomes between cases and controls with a preoperative stay of more than one day. The Mann-Whitney U test was used to compare the postoperative length of stay (secondary outcome) of the patients in the two groups. The Wilcoxon Rank test was used to compare the Borg score between the fourth and the second postoperative days. SPSS (SPSS version 22 Armonk, NY: IBM Corp) was used for performing the statistical analysis. A P value of less than 0.05 was considered to be statistically significant.

\section{Results}

\section{Participants and descriptive data}

A total of 30 consecutive cases and 30 controls participated in the study between October 2011 and October 2013. There were no clinically or statistically significant differences between the two groups in the demographic characteristics, smoking status, ASA grade, the type of surgeries or the administration of epidural analgesia as shown in Table 1. (Appendix 2 for details of surgeries).

All participants in the intervention group participated in the exercise program with the pedal exerciser and the rating of perceived exertion on the Borg scale along with their vital signs were recorded from postoperative day 2 till day 4 . All the patients in this group exercised for a mean of 10 min everyday without experiencing adverse events during and after the session. The vital signs of the patients remained stable both before and after the exercise each day as shown in Table 2. 
Table 1 - Demographic and operative characteristics of controls and cases.

\begin{tabular}{llll} 
Characteristic & \multicolumn{1}{c}{ Cases } & Control & P value \\
\hline Age (Mean, Stdev) years & $61.13(13.79)$ & $61(14.9)$ & 0.832 \\
M: F Ratio & $17: 13$ & $18: 12$ & 0.793 \\
Smoker/Ex smoker: Non smoker & $13: 17$ & $11: 19$ & 0.82 \\
ASA Grade & & & 0.065 \\
I & 2 & 8 & \\
II & 20 & 17 & \\
III & 8 & 5 & \\
IV & 0 & 0 & 0.663 \\
Complexity Of surgery & & & \\
Level1 & 0 & 0 & \\
Level2 & 0 & 2 & \\
Level3 & 25 & 25 & \\
Level4 & 5 & 3 & \\
Level5 & 0 & 0 & 0.113 \\
Epidural analgesia given & 15 & 9 & \\
\hline a Surgical complexity grading and details (Appendix 1 and 2).
\end{tabular}

\section{Outcome}

\section{Primary outcome measures}

The overall incidence of Respiratory Tract Infection in this study was $30 \%$. A total of $5(16.7 \%)$ of the 30 patients in the intervention group and 13 (43.33\%) of 30 patients in the control group developed a RTI. Based on the data, the point estimate of the difference between the rates of RTI in the two groups is $26.66 \%$, favouring the intervention group. The $95 \%$ confidence interval for this difference was $(0.0447,0.488)$, hence the true difference in the rate of RTI in the population lies anywhere between $4.7 \%$ and $48.8 \%$. This difference was statistically significant ( $\mathrm{P}=0.024$; Chi-Square) (Table 3, Fig. 2).

None of the patients in either of the two groups developed a Deep Vein Thrombosis (DVT) or Pulmonary Embolus (PE).

\section{Secondary outcome measures}

The secondary outcome as measured by the Borg score in the cases group showed a decline in the subjective breathlessness at rest on postoperative day $4(\mathrm{P}=0.002$, Wilcoxon Signed Rank Test) (Fig. 3). There was typically a reduction in the BORG values between the day 4 and day 2 of 0.533 units with a $95 \%$ confidence interval of $(0.227,0.839)$.

The median duration of postoperative hospitalisation was 8.5 days (range, 2-28 days) in the intervention group and 11 days (range, 0-52 days) in the control group. KolmogorovSmirnov test showed that the variable across cases and controls did not assume a normal distribution (Fig. 4); hence Mann-Whitney U test was used to compare LOS between the

Table 2 - Exercise parameters for cases on postoperative days (pod) 2, 3 and 4 .

\begin{tabular}{lccr} 
Parameter & Pod 2 & Pod 3 & pod4 \\
\hline Borg Median (P = 0.002) & 3 & 3 & 2 \\
HR(Median)/Min & 92 & 87 & 120 \\
RR(MEDIAN)/Min & 20 & 20 & 20 \\
O2sats(Median) \% & 96.5 & 96 & 96 \\
Time (Median) Min & 10 & 10 & 10 \\
\hline
\end{tabular}

Table 3 - Primary outcome comparison between cases and controls.

\begin{tabular}{|c|c|c|c|}
\hline Primary outcome & Cases & Controls & $P$ value \\
\hline RTI $^{\mathrm{b}}$ Diagnosed (No) & 5 & 13 & 0.024 \\
\hline New/changed Chest X-ray (No.) & 8 & 17 & \\
\hline $\mathrm{WBC}^{\mathrm{a}}>12$ (No.) & 5 & 10 & \\
\hline Temperature $>38^{\circ}$ Celsius (No.) & 11 & 15 & \\
\hline Sputum positive (No.) & 2 & 6 & \\
\hline Antibiotic treatment (No.) & 10 & 14 & \\
\hline Secondary outcome & & & \\
\hline Preoperative stay $>1$ day & 13 & 13 & \\
\hline RTI in patients with & 2 & 7 & 0.039 \\
\hline Preoperative stay $>1$ day & & & \\
\hline $\begin{array}{l}\text { Postoperative LOS } \\
\text { (Median } \pm \text { Interquartile range) } \\
\text { days }\end{array}$ & $\begin{array}{l}8.5 \pm 5.00 \\
(2-28)\end{array}$ & $\begin{array}{l}11 \pm 7.5 \\
(0-52)\end{array}$ & 0.049 \\
\hline
\end{tabular}

two groups. The difference of LOS between the two groups on an average was 5.83 days, being less in the intervention group. The 95\% confidence interval of the difference in the LOS between the two groups was $0.807-10.86$. Hence the early aerobic activity on an average significantly reduced the LOS in intervention group by between 0.807 and 10.86 days. ( $\mathrm{P}=0.049$, Mann-Whitney $\mathrm{U})$.

The rate of respiratory tract infections was more in the controls with a preoperative hospital stay of more than one day $(2 / 13,15.3 \%)$ as compared to cases with a preoperative hospital stay of more than one day $(7 / 13,53.8 \%)(P=0.039$; Fischer's test) (Table 3, Fig. 5). The 95\% confidence interval for this difference was $(0.05,0.719)$. Hence the point estimate of reduction in the RTI in both groups with preoperative stay of $>1$ day was $38.46 \%$ and the difference could be anywhere between $5 \%$ and $71.9 \%$.

There was no statistical difference between the two groups with regards to smoking status or administration of epidural analgesia (Table 1).

\section{Discussion}

Pulmonary complications along with increased morbidity and mortality following surgery ${ }^{2}$ are associated with significant increase in the length of hospital stay and additional health care costs. $^{11}$

A patient safety summit on pulmonary complications calculated the economic burden of PPC by extrapolating data from a study by Linde-Zwirble et al. ${ }^{12}$ PPCs added $\$ 717$ to the average cost of elective surgery with 92,200 additional ICU admissions, 584,300 additional ICU days, and $\$ 3.42$ billion in additional costs. ${ }^{13}$ Thompson et al. $^{14}$ found that PP in abdominal surgical patients increased hospital LOS by 11 days and hospital charges by $\$ 31,000$ (year 2000 dollars). Overall, the mean increase in hospital LOS specifically attributable to PPC is approximately 8 days. ${ }^{6}$

We found that moderate intensity lower extremity aerobic exercise on a stationary pedal exerciser resulted in more than halving the rate of postoperative pulmonary complications for 


\section{Rate of RTI in cases and controls}

Groups RTI

\section{Cases \\ Controls \\ 品O RT}

$\mathbf{p}=\mathbf{0 . 0 2 4}$
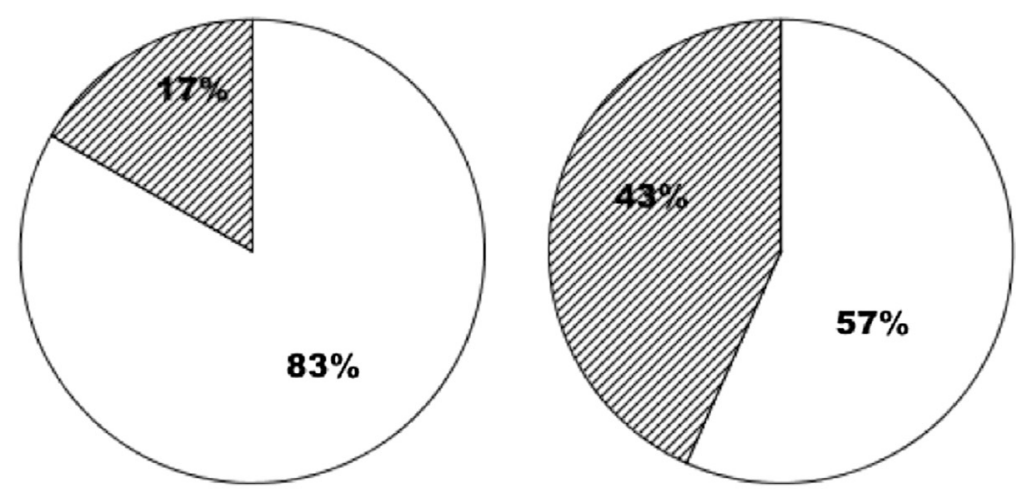

Cases weighted by RATES

Fig. 2 - Pie charts comparing the primary outcome between the two groups.

the exercise group. This reduction of PPC in the population with use of the exercise program could be anywhere between $4.47 \%$ and $48.8 \%$ in the population. (point estimate $26.66 \%$ ).

The intervention group typically had a lesser postoperative length of stay (LOS) as compared to standard postoperative respiratory management. This reduction in average LOS could be between 0.807 and 10.86 days (mean 5.86 days) in the population. The patients (cases and controls) who were admitted preoperatively for longer durations i.e. more than one day had significantly lower rates of respiratory tract infection on an average if they undertook the exercise program. The rate of RTI in this population with the help of the exercise program can be reduced anywhere between $5 \%$ and $71.9 \%$ (point estimate $38.4 \%$ ).

The respiratory function (respiratory muscle force) of the intervention group as measured by the subjective breathlessness on the Borg dyspnoea scale also progressively

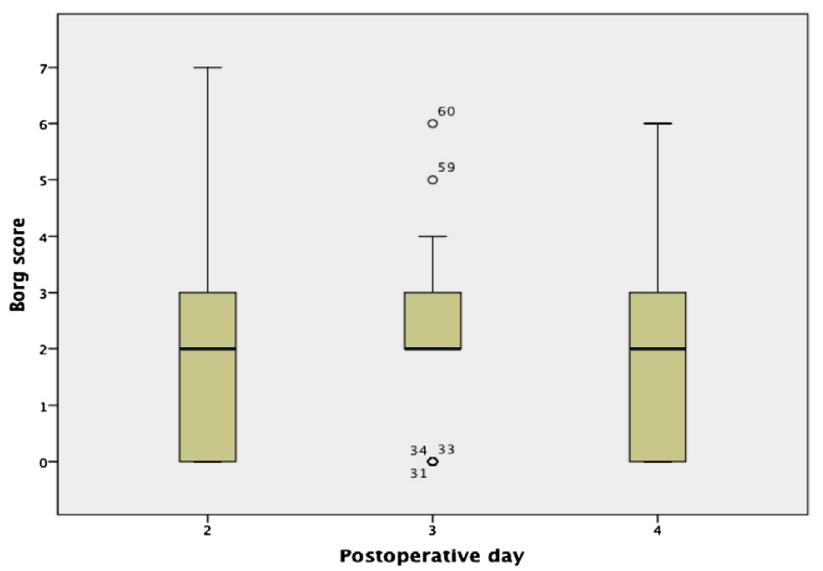

Fig. 3 - Box plot of borg measured over time in the cases $(P=0.001)$. improved over the postoperative period. No adverse effects of early exercise were detected in any of the patients in the intervention group.

The pathogenesis of PPC involves a postoperative impairment of pulmonary function. This includes a pain induced decrease in pulmonary mechanics and neurally reflex mediated inhibition of diaphragmatic function. Also various humoral cascade systems, arachidonic acid metabolism, cytokines, endothelial adhesion factors etc may contribute. In addition, routine postoperative care involving the supine position for several hours daily may impair the pulmonary mechanics and oxygenation. So far, a single standardised technique is not available to improve postoperative pulmonary function and prevent pulmonary complications. ${ }^{15,16,20,21}$

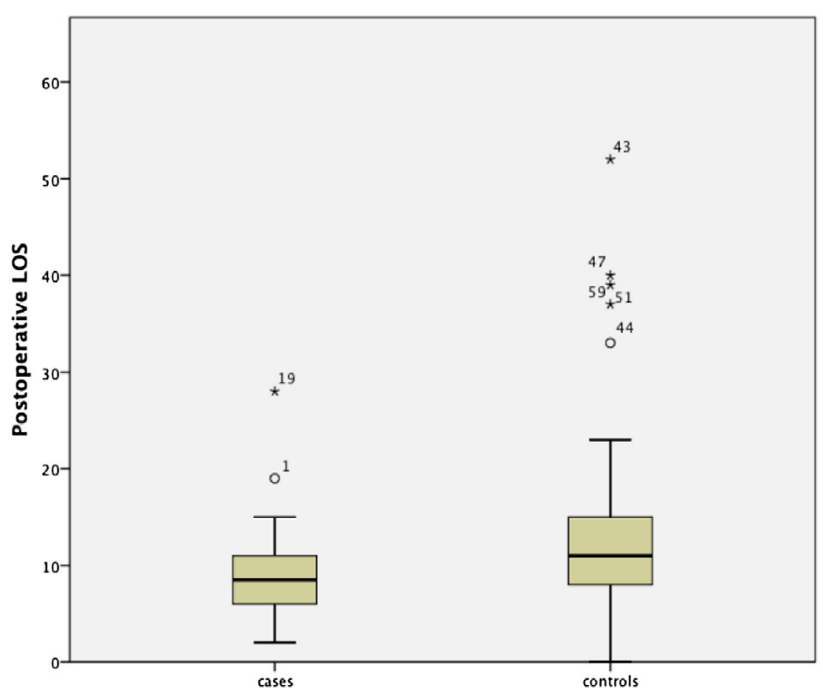

Fig. 4 - Box plot of postoperative length of stay in cases and controls $(P=0.023)$. 


\section{Rate of RTI in cases and controls with a preoperative stay $>1$ day Groups \\ Cases Controls $\quad$ Q}

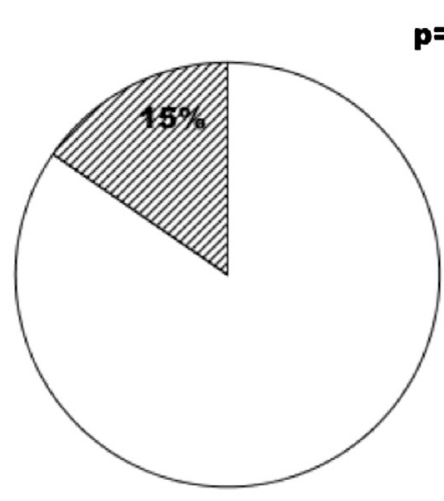

$\mathbf{p}=\mathbf{0 . 0 3 9}$

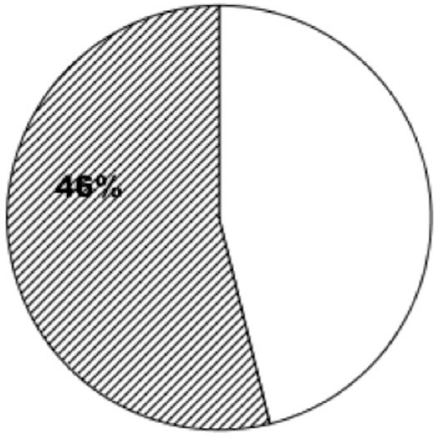

Cases weighted by RATES

Fig. 5 - Pie charts comparing the RTI in patients with preoperative stay of more than one day.

Exercise regimens used perioperatively have shown to improve pulmonary functions patients undergoing thoracic surgery, this group is very different from patients undergoing abdominal surgery and hence the comparisons should be made carefully. A systematic review on perioperative physiotherapy for patients undergoing lung cancer resection concluded that "pre-operative interventions based on moderate-to-intense aerobic exercise in patients undergoing lung resection for lung cancer improve functional capacity and reduce postoperative morbidity, whereas interventions performed only during the postoperative period do not seem to reduce PPCs or LOS." 16 But the postoperative studies included in this review utilised techniques like incentive spirometry, IPPV, breathing and coughing exercises; none of the which used postoperative aerobic exercise while the preoperative studies that reported a reduction in LOS and PPC employed strength and endurance training through aerobic exercise. ${ }^{17,18}$ The authors state, "No firm conclusions can be drawn because of the heterogeneity of the studies analysed". A randomised controlled trial published in 2014, on patients who underwent lung cancer surgery employed high-intensity endurance and strength training (60 min, three times a week, 20 weeks), starting 5-7 weeks after surgery in the intervention group and found that it was well tolerated and led to significant improvements in peak oxygen uptake muscular strength, total muscle mass, functional fitness and quality of life. ${ }^{19}$ The exercise regime employed in this study was longer in duration and higher in intensity as that in our study, but it is difficult to directly compare the two study groups. But it is safe to say that aerobic exercise whether done preoperatively or postoperatively plays some role in reduction of PPC and LOS.

True efficacy of the different techniques presently used is not known because there is a wide variation in the rehabilitation techniques employed, the definitions used, the outcomes measured and the incidence of the PPC. The systematic review by Guimaraes et al. found no evidence regarding the effectiveness of the use of incentive spirometry for prevention of postoperative pulmonary complications in upper abdominal surgery. ${ }^{20}$ They analysed 11 randomised controlled trials for this study. Another systematic review analysing 35 articles concluded that respiratory physiotherapy is not effective in preventing pulmonary complications after abdominal surgery. ${ }^{21}$

Early aerobic activity with the pedal exerciser results in reduction of respiratory morbidity postoperatively; most likely by improving in the pulmonary function. This is in agreement with a recently published article, which states that aerobic exercise when associated with incentive spirometry helps in controlling postoperative pulmonary complications after laparoscopic cholecystectomy ${ }^{22}$ The cause of this reduced hospital stay is not known, most likely it is multifactorial. Improvements in overall dyspnoea, functional performance and health status might be responsible.

Longer preoperative stays are a well-known risk factor for PPC. ${ }^{23}$ The improved physiological function resulting for the early aerobic activity is most likely responsible for this outcome.

High-intensity exercise training near maximal workload follows sound physiologic principles and has established effectiveness in pulmonary rehabilitation in COPD patients. Moderate to high intensity lower limb exercise leads to improvement in peak oxygen consumption $\left(\mathrm{VO}_{2} \mathrm{max}\right)$ and maximal exercise workload. This along with reduced symptoms of dyspnoea and fatigue contribute to the improved respiratory function. ${ }^{24}$

The study has limitations mainly based around the pragmatic study design. As a case-control study, randomisation of the patients was not carried out, so there is a potential for observer bias. To minimise this, the controls were case mix matched prospectively from a similar general surgical service not using postoperative exercising. As illustrated in Table 1, the demographic characteristics, co morbidities, smoking status, administration on epidural analgesia and the type of surgery between the two groups were not significantly different. All cases/controls were administered antibiotic prophylaxis and analgesia as per standard hospital protocols. 
We found that early aerobic activity with a pedal exerciser significantly reduced the rate of postoperative respiratory tract infections, the postoperative length of stay and the perceived breathlessness in the intervention group as compared to the controls. This can be explained by the physiological benefits of a high intensity exercise program, which results in the improvement of pulmonary function, exercise endurance and the overall health status of the patients. Since postoperative high intensity aerobic exercise can lead to early recovery in patients undergoing major surgery it can be considered for the Enhanced Recovery After Surgery (ERAS) pathway. ${ }^{25}$ Further large-scale randomised studies with physiologic testing may be required to see if early aerobic activity is better than the existing rehabilitation techniques for reduction in postoperative pulmonary complications.

\section{Sources of funding}

The project was supported by an unrestricted educational grant from MSD (Merck Sharp \& Dohme).

\section{Appendix 1}

\section{Levels of surgical complexity}

Adapted form L. R. Pasternak: Screening Patients- Strategies and Studies. In Ambulatory Anesthesiology- A problemoriented approach. Ed. By Kathryn McGoldrick. Williams and Wilkins 1995.

Level 1

- Minimal risk to the patient independent of anesthesia

- Minimally invasive procedures with little or no blood loss

- Often done in an office setting with the operating room principally for anesthesia and monitoring

Includes: Breast biopsy, removal of minor skin or subcutaneous lesions, myringtomy tubes, hysteroscopy, cystoscopy, fiberoptic bronchoscopy.

Level 2

- Minimal to moderately invasive procedure

- Blood loss less than 500 cc

- Mild risk to patient independent of anesthesia

Includes: Diagnostic laparoscopy, dilatation, and curettage, fallopian tubal ligation, arthroscopy, inguinal hernia repair, laparoscopic lysis of adhesions, tonsillectomy/adenoidectomy, umbilical hernia repair, septoplasty/rhinoplasty, percutaneous lung biopsy, expensive superficial procedures.

Level 3

- Moderate to significantly invasive procedure

- Blood loss potential 500-1,500 cc

- Moderate risk to patient independent of anesthesia

Includes: hysterectomy, myomectomy, cholecstectomy, laminectomy, hip/knee replacement, major laparscopic procedures, resection/reconstructive surgery of the digestive tract.

Excludes: Open thoracic or intracranial procedure.

Level 4

- Highly invasive procedure

- Blood loss greater than 1,500 cc

- Major risk to patient independent of anesthesia

Includes: Major orthopedic-spinal reconstruction, major reconstruction of the gastrointestinal tract, major vascular repair without postoperative ICU stay.

Level 5

- Highly invasive procedure

- Blood loss greater than 1,500 cc

- Critical risk to patient independent of anesthesia

- Usual postoperative ICU stay with invasive monitoring

Includes: Cardiothoracic procedure, intracranial procedure, major procedure on the oropharynx, major vascular skeletal, neurologic repair.

\section{Appendix 2}

\begin{tabular}{lcc}
\hline Details of surgeries performed & & \\
\hline Type of surgery (P = 0.455) & Cases & Controls \\
\hline $\begin{array}{l}\text { Hepatobiliary e.g. liver resection, biliary } \\
\text { bypass }\end{array}$ & 2 & 2 \\
$\begin{array}{l}\text { Cholecystectomy (laparoscopic/open) } \\
\text { Oesophagogastric } \pm \text { anastomosis (e.g. } \\
\text { gastrectomy, oesophegogastrectomy) }\end{array}$ & 6 & 4 \\
$\begin{array}{l}\text { Colorectal (hemicolectomy, anterior } \\
\text { resection) }\end{array}$ & 4 & 12 \\
$\begin{array}{l}\text { Small bowel (small bowel resection) } \\
\text { Other (diaphragmatic hernia repair, } \\
\text { adrenelectomy, splenectomy) }\end{array}$ & 1 & 1 \\
\hline
\end{tabular}

\section{R E F E R E N C E S}

1. Chumillas SPJ, Delgado F, Viciano V, Mateu M. Prevention of postoperative pulmonary complications through respiratory rehabilitation: a controlled study. Arch Phys Med Rehabil 1998;79:5-9.

2. Arozullah AM, Henderson WG, Khuri SF, Daley J. Postoperative mortality and pulmonary complication rankings: how well do they correlate at the hospital level? Med Care 2003;41(8):979-91.

3. Martin LF, Asher EF, Casey JM, Fry DE. Postoperative pneumonia. Determinants of mortality. Archives Surg 1984;119(4):379-83.

4. Kips JC. Preoperative pulmonary evaluation. Acta Clin Belg 1997;52(5):301-5.

5. Celli BR, Rodriguez KS, Snider GL. A controlled trial of intermittent positive pressure breathing, incentive spirometry, and deep breathing exercises in preventing pulmonary complications after abdominal surgery. Am Rev Respir Dis 1984;130(1):12-5. 
6. Lawrence VA, Cornell JE, Smetana GW, American College of Physicians. Strategies to reduce postoperative pulmonary complications after noncardiothoracic surgery: systematic review for the American college of physicians. Ann Intern Med 2006;144(8):596-608.

7. Pasternak LR. Screening patients-Strategies and studies. In: McGoldrick K, editor. Ambulatory anesthesiology-A problemoriented approach. Williams and Wilkins; 1995.

8. B GA. Psychophysical bases of perceived exertion. Med Sci Sports Exerc 1982;14:377-81.

9. B G. Perceived exertion as an indicator of somatic stress. Scand J Rehabil Med 1970;2:92-8.

10. Ernesto Crisafulli EMC. Measures of dyspnea in pulmonary rehabilitation. Multidiscip Respir Med 2010;5(3):202-10.

11. Lawrence VA, Hilsenbeck SG, Mulrow CD, Dhanda R, Sapp J, Page CP. Incidence and hospital stay for cardiac and pulmonary complications after abdominal surgery. J General Intern Med 1995;10(12):671-8.

12. Linde-Zwirble WL, Bloom JD, Mecca RS, Hansell DM. Postoperative pulmonary complications in adult elective surgery patients in the US: severity, outcomes, and resource use. Crit Care 2010;14(1):210.

13. Shander A, Fleisher LA, Barie PS, Bigatello LM, Sladen RN, Watson CB. Clinical and economic burden of postoperative pulmonary complications: patient safety summit on definition, risk-reducing interventions, and preventive strategies. Crit Care Med 2011;39(9):2163-72. Epub 2011/05/17.

14. Thompson DA, Makary MA, Dorman T, Pronovost PJ. Clinical and economic outcomes of hospital acquired pneumonia in intra-abdominal surgery patients. Ann Surg 2006;243:547-52.

15. Kehlet H. Multimodal approach to control postoperative pathophysiology and rehabilitation. $\mathrm{Br} J$ Anaesth 1997;78:606-17.

16. Rodriguez-Larrad A, Lascurain-Aguirrebena I, AbeciaInchaurregui LC, Seco J. Perioperative physiotherapy in patients undergoing lung cancer resection. Interact Cardiovasc Thorac Surg 2014;19(2):269-81.
17. Benzo R, Wigle D, Novotny P, Wetzstein M, Nichols F, Shen RK, et al. Preoperative pulmonary rehabilitation before lung cancer resection: results from two randomized studies. Lung Cancer 2011;74(3):441-5.

18. Morano MT, Araújo AS, Nascimento FB, da Silva GF, Mesquita R, Pinto JS, et al. Preoperative pulmonary rehabilitation versus chest physical therapy in patients undergoing lung cancer resection: a pilot randomized controlled trial. Archives Phys Med Rehabil 2013;94(1):53-8.

19. Edvardsen E, Skjonsberg OH, Holme I, Nordsletten L, Borchsenius F, Anderssen SA. High-intensity training following lung cancer surgery: a randomised controlled trial. Thorax 2015 Mar;70(3):244-50.

20. Guimaraes MM, El Dib R, Smith AF, Matos D. Incentive spirometry for prevention of postoperative pulmonary complications in upper abdominal surgery. Cochrane Database Syst Rev 2009;8(3). CD006058.

21. Pasquina P, Tramer MR, Granier JM, Walder B. Respiratory physiotherapy to prevent pulmonary complications after abdominal surgery: a systematic review. Chest 2006;130(6):1887-99. Epub 2006/12/15.

22. El-Marakby A, Darwiesh A, Anwar E, Mostafa A, El- Gendy SR, Allah Gaowgzeh R. Aerobic exercise training and incentive spirometry can control postoperative pulmonary complications after laparoscopic cholecystectomy. MiddleEast J Sci Res 2013;13(4):459-63.

23. Garibaldi RA, Britt MR, Coleman ML, Reading JC, Pace NL. Risk factors for postoperative pneumonia. Am J Med 1981;70(3):677-80. Epub 1981/03/01.

24. Normandin EA, McCusker C, Connors M, Vale F, Gerardi D, ZuWallack RL. An evaluation of two Approaches to exercise conditioning in pulmonary rehabilitation. Chest 2002;121:1085-91.

25. ERAS Society. ERAS protocol (EP). 2010-13. http:// wwwerassocietyorg/indexphp/eras-care-system/erasprotocol. 